\title{
DYNAMICAL STABILITY OF FLUID SPHERES IN SPACETIMES WITH A NONZERO COSMOLOGICAL CONSTANT*
}

\author{
STANISLAV HLEDÍK ${ }^{\dagger}$, ZDENĚK STUCHLÍK ${ }^{\ddagger}$ and KRISTINA MRÁZOVÁ $\odot$ \\ Institute of Physics, Faculty of Philosophy and Science, Silesian University in Opava, \\ Bezručovo nám. 13, Opava, CZ-74601, Czech Republic \\ E-mail: ${ }^{\dagger}$ Stanislav.Hledik@fpf.slu.cz, ${ }^{\ddagger}$ Zdenek.Stuchlik@fpf.slu.cz, ${ }^{\odot}$ Kristina.Mrazova@fpf.slu.cz
}

\begin{abstract}
The Sturm-Liouville eigenvalue equation for eigenmodes of the radial oscillations is determined for spherically symmetric perfect fluid configurations in spacetimes with a nonzero cosmological constant and applied in the cases of configurations with uniform distribution of energy density and polytropic spheres. It is shown that a repulsive cosmological constant rises the critical adiabatic index and decreases the critical radius under which the dynamical instability occurs.
\end{abstract}

Keywords: Perfect fluid configurations; Cosmological constant; Dynamical stability.

\section{Introduction}

The internal Schwarzschild spacetimes with nonzero cosmological constant $(\Lambda \neq 0)$ and uniform distribution of energy density were given for star-like configurations ${ }^{1}$ and extended to more general situations. ${ }^{2}$ The polytropic and adiabatic spheres were preliminary treated and compared, ${ }^{3,4}$ neutron star models with regions of nuclear matter described by different relativistic equations of state that are matched were also treated. ${ }^{5}$ Their stability can be considered on energetic grounds ${ }^{6}$ or it can be treated in dynamical way. ${ }^{7}$ Here we determine the dynamical stability conditions for the uniform density and polytropic spheres using the approach of Misner et al. ${ }^{8}$

\section{Sturm-Liouville Equation}

Interior of a spherically symmetric configuration is described (in standard Schwarzschild coordinates) by the line element

$$
\mathrm{d} s^{2}=-\mathrm{e}^{2 \Phi} \mathrm{d} t^{2}+\mathrm{e}^{2 \Psi} \mathrm{d} r^{2}+r^{2}\left(\mathrm{~d} \theta^{2}+\sin ^{2} \theta \mathrm{d} \varphi^{2}\right),
$$

with metric coefficients taken in the general form

$$
\Psi=\Psi(r, t), \quad \Phi=\Phi(r, t) .
$$

The perfect fluid distribution is given by energy density and pressure profiles $\rho(r, t)$ and $p(r, t)$. The static equilibrium configuration is given by $\Phi_{0}(r), \Psi_{0}(r), \rho_{0}(r)$, $p_{0}(r)$ satisfying the Euler equations. The radially pulsating configuration is then determined by

$$
q(r, t)=q_{0}(r)+\delta q(r, t), \quad \delta q \equiv(\delta \Phi, \delta \Psi, \delta \rho, \delta p, \delta n),
$$

*This research has been supported by Czech grant MSM 4781305903. 
where $n$ is the number density. The pulsation is given by the radial displacement $\xi=\xi(r, t)$. The Euler perturbations $\delta q$ are related to the Lagrangian perturbations measured by an observer who moves with the pulsating fluid by the relation

$$
\Delta q(r, t)=q(r+\xi(r, t), t)-q_{0}(r) \approx \delta q+q_{0}^{\prime} \xi .
$$

Introducing a new variable $\zeta \equiv r^{2} \mathrm{e}^{-\Phi_{0}} \xi$, the radial pulsations are governed by

$$
W \ddot{\zeta}=\left(P \zeta^{\prime}\right)^{\prime}+Q \zeta
$$

with the functions $W(r), P(r), Q(r)$ determined for the equilibrium configuration

$$
\begin{gathered}
W \equiv\left(\rho_{0}+p_{0}\right) \frac{1}{r^{2}} \mathrm{e}^{3 \Psi_{0}+\Phi_{0}}, \\
P \equiv \gamma p_{0} \frac{1}{r^{2}} \mathrm{e}^{\Psi_{0}+3 \Phi_{0}}, \\
Q \equiv \mathrm{e}^{\Psi_{0}+3 \Phi_{0}}\left[\frac{\left(p_{0}^{\prime}\right)^{2}}{\rho_{0}+p_{0}} \frac{1}{r^{2}}-\frac{4 p_{0}^{\prime}}{r^{3}}-\left(\rho_{0}+p_{0}\right)\left(\frac{8 \pi G}{c^{4}} p_{0}-\Lambda\right) \frac{\mathrm{e}^{2 \Psi_{0}}}{r^{2}}\right] .
\end{gathered}
$$

The linear stability analysis can be realized by the standard assumption of the displacement decomposition

$$
\zeta(r, t)=\zeta(r) \mathrm{e}^{\mathrm{i} \omega t} .
$$

Then the dynamic equation reduces to the Sturm-Liouville equation and the related boundary conditions in the form

$$
\begin{gathered}
\left(P \zeta^{\prime}\right)^{\prime}+\left(Q+\omega^{2} W\right) \zeta=0, \\
\frac{\zeta}{r^{3}} \quad \text { finite as } r \rightarrow 0, \quad \gamma p_{0} \frac{\mathrm{e}^{\Phi_{0}}}{r^{2}} \zeta^{\prime} \rightarrow 0 \quad \text { as } \quad r \rightarrow R .
\end{gathered}
$$

The Sturm-Liouville equation (10) and the boundary conditions determine eigenfrequencies $\omega_{j}$ and corresponding eigenmodes $\zeta_{i}(r)$, where $i=1,2, \ldots, n$. The eigenvalue Sturm-Liouville (SL) problem can be expressed in the variational form ${ }^{8}$ as the extremal values of

$$
\omega^{2}=\frac{\int_{0}^{R}\left(P \zeta^{\prime 2}-Q \zeta^{2}\right) \mathrm{d} r}{\int_{0}^{R} W \zeta^{2} \mathrm{~d} r}
$$

determine the eigenfrequencies $\omega_{i}$ and the corresponding functions $\zeta_{i}(r)$ are the eigenfunctions that have to satisfy the orthogonality relation

$$
\int_{0}^{R} \mathrm{e}^{3 \Psi_{0}-\Phi_{0}}\left(p_{0}+\rho_{0}\right) r^{2} \xi^{(i)} \xi^{(j)} \mathrm{d} r=0 .
$$

\section{Results and Conclusions}

We applied the Sturm-Liouville approach to spheres with uniform energy density, ${ }^{1}$ and polytropic spheres. ${ }^{3}$ The case of uniform spheres can be properly taken as a test bed of the dynamical instability problem - although these solutions of the Einstein equations are of rather artificial character, they reflect quite well the basic properties 


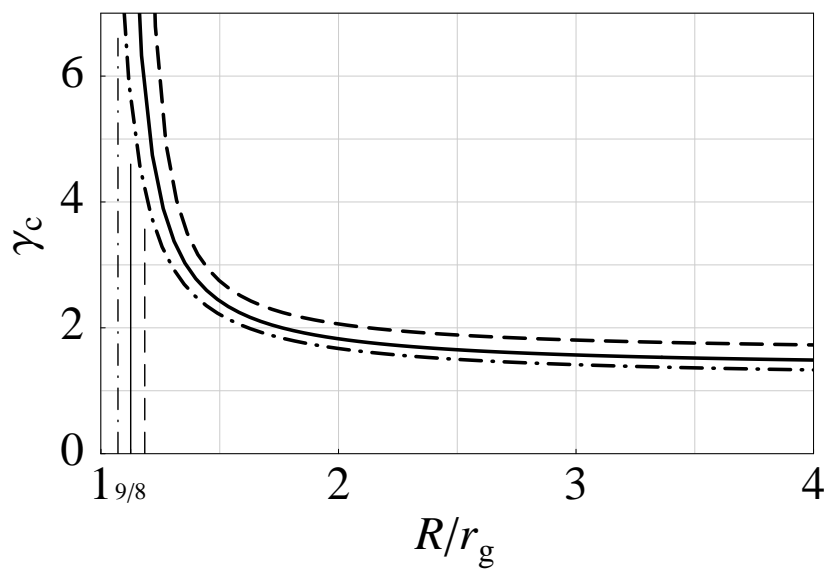

Figure 1. Dependence of the critical value of adiabatic index $\gamma_{\mathrm{c}}$ on sphere radius $R$. Full curve: vanishing cosmological constant $\lambda=0$; then $\gamma_{\mathrm{c}}$ diverges as $R \rightarrow 9 r_{\mathrm{g}} / 8$ from above. Dashed curve: positive cosmological constant $\lambda=0.1$, the point of divergence is shifted to $1.18421>9 / 8$, and $\gamma_{\mathrm{c}, \lambda>0}>\gamma_{\mathrm{c}, \lambda=0}$. Dashed-dotted curve: negative cosmological constant $\lambda=-0.1$, the point of divergence is shifted to $1.07143<9 / 8$, and $\gamma_{\mathrm{c}, \lambda<0}<\gamma_{\mathrm{c}, \lambda=0}$.

of very compact objects. ${ }^{9}$ The dependence of the critical value of adiabatic index $\gamma \equiv(\partial \ln p / \partial \ln n)_{s}=(n / p)(\Delta p / \Delta n)$ on configuration radius $R$ is for the uniform case given in Fig. 11 (see also ${ }^{12}$ ). The polytropic case is treated in details by Stuchlík and Hledík. ${ }^{10}$

\section{Bibliography}

1. Z. Stuchlík, Acta Phys. Slovaca 50, 219 (2000).

2. C. G. Böhmer, Gen. Relativity Gravitation 36, p. 1039 (2004).

3. Z. Stuchlík and S. Hledík, General Relativistic Polytropes with a Nonzero Cosmological Constant, In preparation, (2006).

4. S. Hledík, Z. Stuchlík and K. Mrázová, General relativistic adiabatic fluid spheres with a repulsive cosmological constant, In preparation, (2006).

5. M. Urbanec, J. Miller and J. R. Stone, Matching of equations of state: influence on calculated neutron star models pp. $357-362$. In Hledík and Stuchlík. ${ }^{11}$

6. R. F. Tooper, Astrophys. J. 140, 434 (1964).

7. S. Chandrasekhar, Astrophys. J. 140, p. 417 (1964).

8. C. W. Misner, K. S. Thorne and J. A. Wheeler, Gravitation (Freeman, San Francisco, 1973).

9. N. K. Glendenning, Phys. Rev. C 37, p. 2733 (1988).

10. Z. Stuchlík and S. Hledík, Radial pulsations and dynamical stability of spherically symmetric perfect fluid configurations in spacetimes with a nonzero cosmological constant pp. 209-222. In Hledík and Stuchlík. ${ }^{11}$

11. S. Hledík and Z. Stuchlík (eds.), Proceedings of RAGtime 6/r7: Workshops on black holes and neutron stars, Opava, 16-18/18-20 September 2004/2005 (Silesian University in Opava, Opava, 2005).

12. C. G. Böhmer and T. Harko, Dynamical instability of fluid spheres in the presence of a cosmological constant, Phys. Rev. D 71, p. 084026 (2005), arXiv: gr-qc/0504075 